\title{
Cerebral and muscle near-infrared spectroscopy during lower-limb muscle activity - volitional and functional electrical stimulation
}

Anirban Dutta ( $\square$ anirband@buffalo.edu )

University at Buffalo SUNY

Fei Zhao

University at Buffalo SUNY, USA

Mancheung Cheung

University at Buffalo SUNY, USA

Abhijit Das

The Walton Centre NHS Foundation Trust, UK

Machiko Tomita

University at Buffalo SUNY, USA

Kausik Chatterjee

Countess of Chester Hospital NHS Foundation Trust, Chester, UK

\section{Method Article}

Keywords: Chronic venous insufficiency, functional electrical stimulation, fNIRS, EEG, EMG

Posted Date: July 20th, 2021

DOl: https://doi.org/10.21203/rs.3.rs-730088/v1

License: (9) (i) This work is licensed under a Creative Commons Attribution 4.0 International License.

Read Full License 


\title{
Cerebral and muscle near-infrared spectroscopy during lower-limb muscle activity - volitional and functional electrical stimulation
}

\author{
Anirban Dutta, Fei Zhao, Mancheung Cheung, Abhijit Das, Machiko Tomita, and Kausik Chatterjee
}

\begin{abstract}
Chronic venous insufficiency (CVI) can lead to blood clotting in the deep veins of the legs, a disease known as deep vein thrombosis. An estimated 40 percent of people in the United States have venous insufficiency that may be ameliorated with functional electrical stimulation (FES). Near-infrared spectroscopy (NIRS) is a non-invasive optical imaging method for monitoring hemodynamics. NIRS being an optical technique can be combined with FES for a theranostics application without suffering stimulation artefact. In this study, we combined muscle NIRS with electromyogram (EMG) of the calf muscles to detect blood volume changes (based on total hemoglobin concentration) in the muscle during volitional tiptoe movements at different frequencies as well as during FES (using $g e k o^{\mathrm{TM}}$ ) with different stimulation intensity. In muscle NIRS+FES study, we also measured the cerebral hemodynamics using functional NIRS (fNIRS). Muscle NIRS was conducted using a frequency domain (FD) method (called FDNIRS) that used a multi-distance method to isolate muscle hemodynamics. FDNIRS-EMG study in ten healthy humans found a statistically significant $(p<0.05)$ effect of the tiptoe frequencies on the EMG magnitude (and power) that increased with tiptoe frequency. Also, the muscle blood volume (standing/rest) decreased $\mathbf{p}<\mathbf{0 . 0 1}$ ) with increasing tiptoe frequency and increasing stimulation intensity that was statistically significantly $(p<0.05)$ different for males and females. Moreover, increasing stimulation intensity led to a statistically significant $(p<0.01)$ increase in cerebral blood volume measured with fNIRS. Therefore, combined muscle NIRS and fNIRS with FES can provide a theranostics application in CVI.
\end{abstract}

\section{INTRODUCTION}

Near-infrared spectroscopy (NIRS) is a non-invasive optical imaging method for monitoring oxygen availability and utilization in the tissues [1]. NIRS allows relative measurements of changes in the hemoglobin oxygenation in the brain and hemoglobin plus myoglobin oxygenation changes in the muscle during a functional task. Muscle NIRS has been performed for lower limb muscles during diverse conditions [2]. Also, functional NIRS (fNIRS) of the brain has undergone consistent improvements and its application has increased drastically over the years [3].

In this paper, we applied multi-distance frequency domain (FD) NIRS to study muscle hemodynamics and continuouswave $(\mathrm{CW})$ fNIRS to study cerebral hemodynamics during lower-limb tip-toe exercise and functional electrical stimulation (FES) of the common peroneal nerve using $g e k o^{\mathrm{TM}}$. In FD NIRS, the light intensity is modulated at radio

"Research supported by University at Buffalo SUNY.

A. Dutta is with is with the dept. of Biomedical Engineering, University at Buffalo SUNY, Buffalo, NY 14226; (e-mail: anirband@buffalo.edu).

F. Zhao is with the dept. of Rehabilitation Science, University at Buffalo SUNY, Buffalo, NY 14226; (e-mail: fzhao6@buffalo.edu).

M. Cheung is with the dept. of Biomedical Engineering, University at Buffalo SUNY, Buffalo, NY 14226; (e-mail: mancheun @ buffalo.edu). frequencies in the range of several $10-100 \mathrm{MHz}$ and then sent through the tissue. The photomultiplier tubes or fast

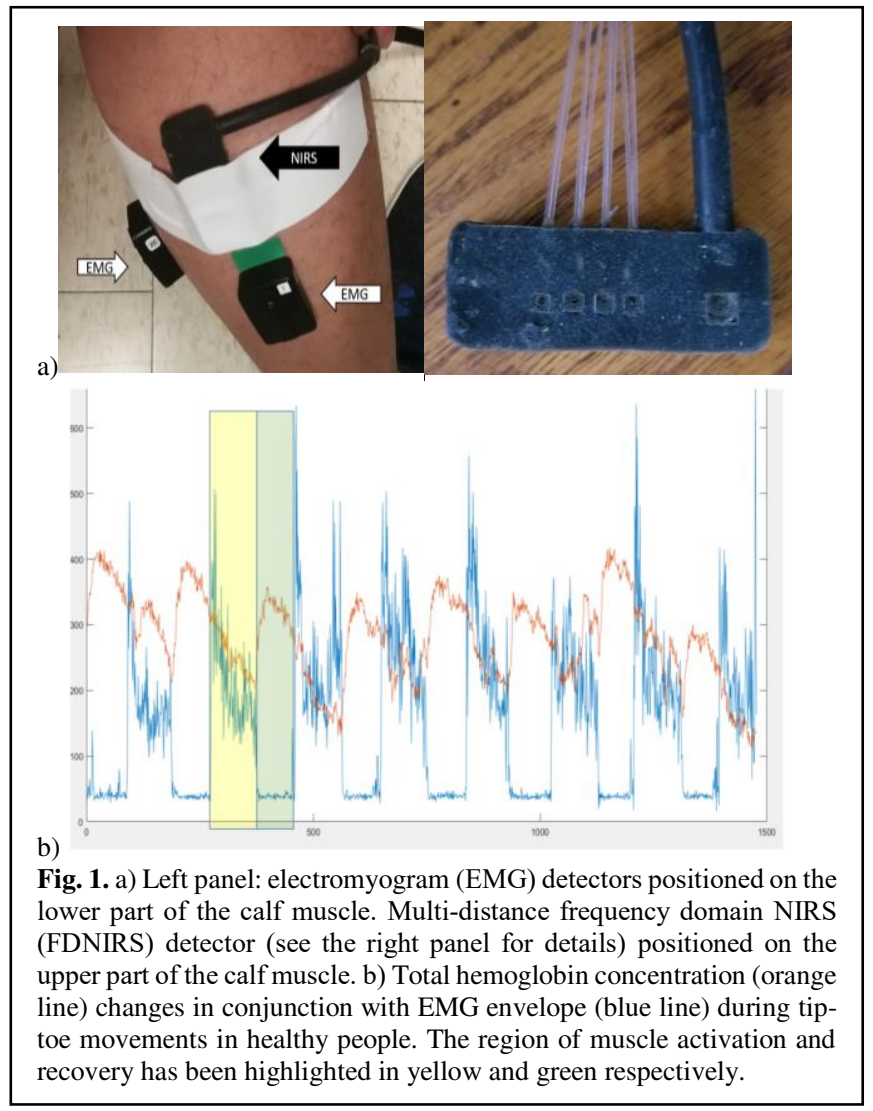

photodiodes collect the scattered light, which shows attenuation as well as a phase shift with respect to the incident signal. The measurement of phase shift allows the calculation of the optical path length and thereby differentiates between scattering and absorption effects. In CW fNIRS, continuous light is used to non-invasively investigate changes in the hemoglobin oxygenation in the human brain tissue [4]. We also combined muscle NIRS with electromyogram (EMG) to better quantify the hemodynamic response during tip-toe exercise in young and healthy humans [5], as shown in Fig. 1.

\section{METHODS}

\section{A. Muscle NIRS-EMG and human study}

The institutional review board and ethics committee of the

A. Das is with The Walton Centre NHS Foundation Trust, UK; (e-mail: abhijit.neuro@gmail.com).

M. Tomita is with the dept. of Rehabilitation Science, University at Buffalo SUNY, Buffalo, NY 14226; (e-mail: Machikot@buffalo.edu).

$\mathrm{K}$. Chatterjee is with the dept. of Stroke Medicine, Countess of Chester Hospital NHS Foundation Trust, Chester, UK; (e-mail: kausikchatterjee@nhs.net). 
university approved the study. Ten young and healthy subjects (age 21-28 years, six males) participated in the study. In order to capture the effects of muscle contraction on hemodynamics, we combined muscle NIRS with EMG of the calf muscles to detect blood volume changes in the muscle based on total hemoglobin concentration (HbT) at different frequency of tiptoe movements and FES. The subjects were provided a metronome (beats per minute, BPM: 10,30,50) to make the tiptoe movements. Fig. 1 shows the multi-distance FDNIRSEMG sensor montage. Here, the muscle NIRS was captured at $10 \mathrm{~Hz}$ using the Imagent ${ }^{\mathrm{TM}}$ FDNIRS system (ISS, USA) and the EMG (DC-500 Hz, $160 \mathrm{~dB} / \mathrm{Dec}$ ) was captured at $2000 \mathrm{~Hz}$ by the Trigno EMG Systems (Delsys, Inc. USA). Multidistance FDNIRS flexible sensor (right panel of Fig. 1) was placed on the skin using a bandage without tightening. Two EMG detectors were placed under the FDNIRS to record the calf muscle activation during the tip-toe movements. Three tip-toe rates, 10, 30, $50 \mathrm{BPM}$, were presented to the subjects in random order. Each subject would be tested twice for 10, 30 and 50 BPM, as listed in the Table I.

In a separate study on ten young and healthy subjects (age 21-28 years, six males), FES was conducted using a disposal $g e k o^{\mathrm{TM}}$ device for transcutaneous peroneal nerve stimulation at 11 preset stimulation levels presented in random order. The 11 preset stimulation levels included pulse-widths of $35 \mu \mathrm{s}, 50 \mu \mathrm{s}, 70 \mu \mathrm{s}, 100 \mu \mathrm{s}, 140 \mu \mathrm{s}, 200 \mu \mathrm{s}$, $280 \mu \mathrm{s}$ at $27 \mathrm{~mA}$ pulse current, then $280 \mu \mathrm{s}$ and $400 \mu \mathrm{s}$ pulse-widths at $38 \mathrm{~mA}$ pulse current, and then $400 \mu \mathrm{s}, 560$ $\mu$ s pulse-widths at $54 \mathrm{~mA} 38 \mathrm{~mA}$ pulse current. After a rest of 5 minutes, the baseline measures were taken for 5 minutes while the subject was seated. Then, FES was applied at eleven stimulation intensities in random order. In repeated-measure design, FES was applied for 2 minute with a rest of 3 minutes while muscle NIRS-EMG and fNIRS were recorded for a duration of 55 minutes with the subject seated with the knee knees bent at 90-degrees.
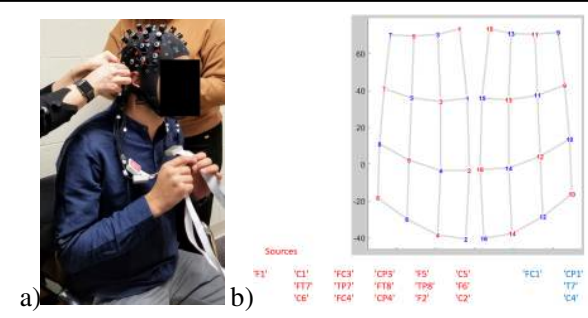

Montage

Fig. 2. a) fNIRS cap setup. b) fNIRS optode montage with sources and detectors placed according to 10/10 electroencephalogram locations.

TABLE I. EXPERIMENTAL PROTOCOL FOR FDNIRS-EMG TEST SUBJECT-Wise BPM ALLOCATION

\begin{tabular}{l|l|l|l|l|l|l} 
Name Mark Gender & Test1 & Test2 & Test3 & Test4 & Test5 & Test6
\end{tabular}

\begin{tabular}{|c|c|c|c|c|c|c|}
\hline Name Mark Gender & Test1 & Test2 & Test3 & Test4 & Test5 & Test6 \\
\hline Subject1 [M] M & 30 & 50 & 10 & 30 & 10 & 50 \\
\hline Subject2 [R] F & 10 & 30 & 50 & 30 & 50 & 10 \\
\hline Subject3 [Z] F & 10 & 30 & 50 & 30 & 50 & 10 \\
\hline Subject4 [W] M & 30 & 50 & 10 & 30 & 10 & 50 \\
\hline Subject5 [B] M & 10 & 30 & 50 & 30 & 10 & 50 \\
\hline Subject6 [Y] F & 30 & 10 & 50 & 30 & 50 & 10 \\
\hline Subject7 [J] M & 30 & 10 & 50 & 10 & 50 & 30 \\
\hline Subject8 [C] F & 30 & 10 & 50 & 30 & 50 & 10 \\
\hline Subject9 [X] M & 10 & 30 & 50 & 30 & 50 & 10 \\
\hline Subject10 [P] M & 10 & 50 & 30 & 50 & 10 & 30
\end{tabular}

B. fNIRS and human study

After fNIRS cap setup (see Fig. 2a), fNIRS was conducted

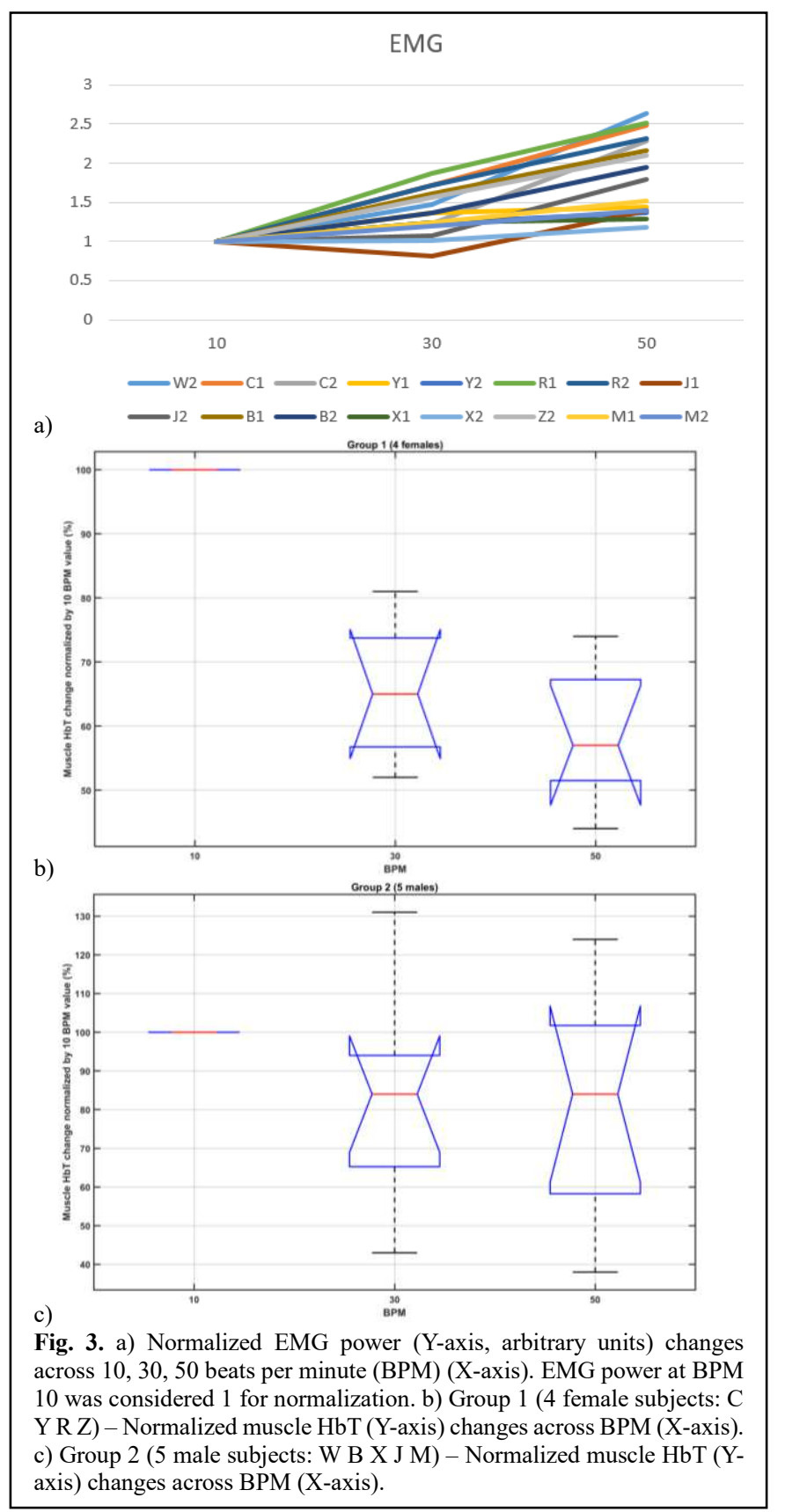

at $10 \mathrm{~Hz}$ using NIRSPORT 2 (NIRx, USA) during eleven repeated blocks of 3 minutes rest and 2 minutes FES for each subject with stimulation intensity applied in random order. Our fNIRS optode montage consisted of 16 long-separation $(\sim 3.5 \mathrm{~cm})$ sources and 16 long-separation $(\sim 3.5 \mathrm{~cm})$ detectors that covered the sensorimotor brain areas as shown in Fig. $2 \mathrm{~b}$.

\section{EMG pre-processing}

EMGworks Analysis (Delsys Inc., USA) was used to compute the root-mean-square EMG envelope, as shown in Fig. 1b. Root-mean-square EMG envelope provided the amplitude of the EMG signal as a measure of the signal power. The volitional EMG envelope was resampled at $10 \mathrm{~Hz}$ to match the sampling frequency of FDNIRS. EMG was not investigated for the FES study due to stimulation artefacts [6]. 


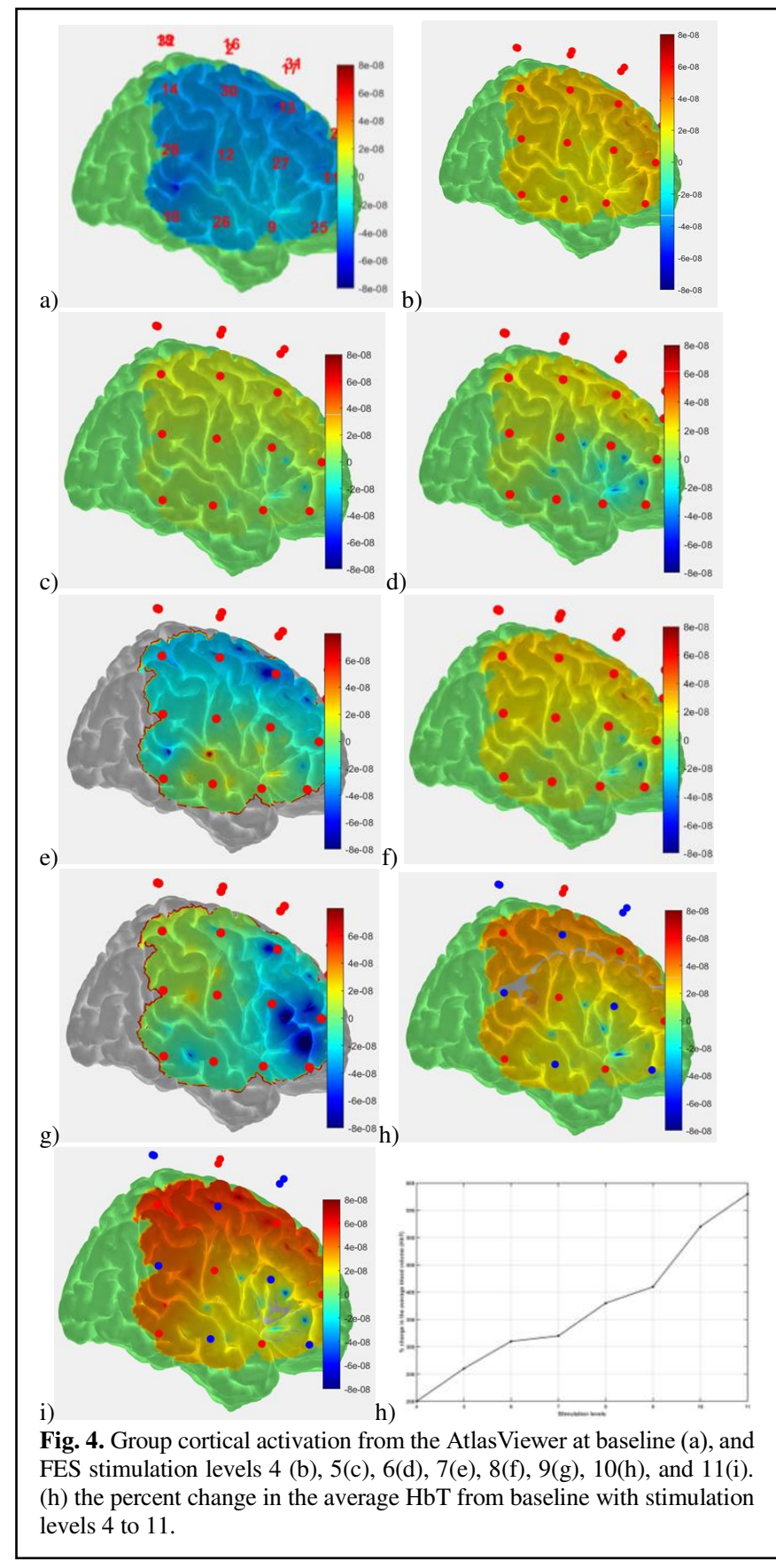

\section{Muscle NIRS-EMG joint-processing}

ISS Boxy Software package (ISS, USA) was used to process the multi-distance FDNIRS data. We computed total hemoglobin concentration $(\mathrm{HbT})$ by adding the oxyhemoglobin and deoxyhemoglobin concentration to estimate the blood volume [7]. Here, the calf venous bloodfilling index (during standing/rest) and calf venous ejection index (after tip-toe movement) are important parameters to be considered during light-intensity exercise for clinical stages of CVI [8]. Therefore, the related regions of muscle activation (tip-toe movement) and recovery (standing/rest) from EMG were epoched in yellow and green respectively as shown in Fig. 1b. At an increased tip-toe rate (10,30, $50 \mathrm{BPM})$, the time available for recovery following calf venous ejection was reduced. All subject data was normalized with the measures at $10 \mathrm{BPM}$ in order to compare the changes in the EMG envelope and average $\mathrm{HbT}$ at recovery epoch for 30 and $50 \mathrm{BPM}$. After normalization with $10 \mathrm{BPM}$, we used two-way ANOVA (analysis of variance) and post-hoc multiple-comparisons ('multcompare' in Matlab) to see if factors - subjects and/or BPM - have an effect. ANOVA compares the means of factors - subjects and/or BPM - to test the hypothesis that they are all equal against the general alternative that they are not all equal.

\section{E. fNIRS processing}

Data processing was conducted using the open-source HOMER3 toolbox [9] in Matlab (Mathworks Inc., USA). The raw optical intensity signal was first converted into optical density (function: hmrR_Intensity2OD), then motion artifact detection and correction was conducted using a hybrid method based on a spline interpolation method and Savitzky-Golay filtering (function: hmrR_MotionCorrectSplineSG) [10] using default parameters. Then, bandpass filtering was conducted (function:hmrR_BandpassFilt:Bandpass_Filter_OpticalDensi ty) within $0.01-0.1 \mathrm{~Hz}$ followed by conversion to oxyhemoglobin ( $\mathrm{HbO}$ ) and deoxy-hemoglobin ( $\mathrm{HHb})$ concentration with default partial path-length factor (function: hmrR_OD2Conc). Finally, short-separation (SS) regression was performed using the average of all long separation channels before computing the hemodynamic response function (HRF) using General Linear Model (GLM) (function: hmrR_GLM_new). GLM was performed to determine the HRF during the stimulation period from the resting state using ordinary least squares [11] with the consecutive sequence of Gaussian functions ( $\mathrm{stdev}=0.5$, step $=0.5$ ). Then, we used open-source AtlasViewer [12] in Matlab (Mathworks Inc., USA) to determine cortical activation for the HRF. We used their default "Colin27" digital brain atlas for HRF image reconstruction with regularization scaling parameter $=0.01$.

\section{RESULTS}

\section{A. Muscle NIRS results}

Fig. 3a shows an increase in the EMG power for increase in BPM, as expected. We conducted two-way ANOVA with factor groups, subject and BPM. The muscle NIRS (HbT) data showed a subject's gender specific effect $(\mathrm{p}<0.05)$, as shown in the Fig. $3 \mathrm{~b}$ and $3 \mathrm{c}$, where the subjects were divided into two groups - female (Group 1) and male (Group 2). Fig. 3b shows the muscle NIRS ( $\mathrm{HbT}$ ) data for the Group 1 and Fig. 3c shows the muscle NIRS (HbT) data for the Group 2. The muscle NIRS (HbT) decreased with an increase in BPM due to reduced time for recovery; however, a floor effect (i.e., a minimum venous retention index) can be seen for higher BPM which is more prominent in male Group 2 than the female Group 1. Fig. $3 \mathrm{~b}$ and $3 \mathrm{c}$ also shows a significant $(\mathrm{p}<0.01)$ effect of BPM. In the FES study, the male and female muscle NIRS (HbT) responded differently $(p<0.05)$, as well as there was a significant $(p<0.01)$ effect of the FES stimulation levels.

\section{B. fNIRS results}

Group cortical activation maps from the AtlasViewer [12] is shown in Fig. 4. There was a significant $(p<0.01)$ effect of the FES stimulation levels. Here, higher stimulation levels led to 
higher cortical activation across the brain when compared to baseline (no FES) as shown in Fig. 4h. However, stimulation levels 7 (Fig. 4e) and 9 (Fig. g) led to regional hypoactivation that are related to $280 \mu \mathrm{s}$ at $27 \mathrm{~mA}$ pulse current (stimulation levels 7) and $400 \mu \mathrm{s}$ pulse-widths at $38 \mathrm{~mA}$ pulse current (stimulation levels 9) respectively.

\section{DISCUSSION}

This preliminary study showed the feasibility of muscle NIRS and fNIRS during volitional lower limb activity and FES. During volitional lower limb tip-toe movements, a floor effect was observed with an increasing BPM (see Fig. 3) where HbT (blood volume) in the muscle could not decrease at the same rate (i.e., a minimum venous retention index) with increasing BPM (or, muscle activation as seen by EMG power - Fig. 3b,c). From the FDNIRS-EMG data, we found that the change in EMG with increasing BPM was similar across subjects, which indicated similar calf muscle activation across male and female subjects. Here, as BPM increased, the EMG power increased almost linearly with BPM where the slopes were found similar across subjects. However, for the muscle NIRS (HbT), HbT decreased more with an increase in BPM in female Group 1 when compared to the male Group 2, as shown in Fig. $3 b$ and 3c. Prior work [13] have shown that males have a higher peak systolic velocity and higher volume flows due to bigger calf muscles and the vein diameters are greater in males than females. Therefore, the difference between the female Group 1 and the male Group 2 can be explained by gender differences in the anatomy - males' calf muscles are bigger, with larger vein diameters than females', therefore, changes during increasing BPM are smaller in males than females due to faster hemodynamic recovery (venous blood-filling) rate. Our gender differences found muscle NIRS-EMG approach during muscle activation feasible for venous sufficiency (venous blood-filling) that is different from person to person according to individual vessel geometry, blood viscosity, etc.

In our prior computational modeling [14], we concluded that in the range of $3.4-4.25 \mathrm{~N}^{*} \mathrm{~s} / \mathrm{m}^{\wedge} 2$ of viscosity and $6-12$ $\mathrm{Hz}$ stimulation frequency, a decrease in the first peak of the blood vessel's end velocity was found with an increase in viscosity and a decrease in pulsatile frequency. This is critical since CVI is a condition that occurs due to dysfunction of the venous wall and/or valves in the leg veins, making it difficult for blood to return to the heart from the legs leading to venous stasis. Blood flow from the periphery to the right atrium is referred to as venous return. This flow back to the heart against gravity during standing is facilitated by lower limb muscle contraction that compresses the local intramuscular and deep veins. This increases the intraluminal pressure that drives this venous flow while retrograde flow is prevented through the action of a system of muscular venous pumps and bicuspid valves. Here, aging and many cardiovascular diseases are related to abnormal cardiac output that may be partly due to inadequate venous return. CVI can lead to blood clotting in the deep veins of the legs, a disease known as deep vein thrombosis, which is also known as post-thrombotic syndrome.

Based on the experimental results, we postulate that muscle NIRS in conjunction with EMG can not only be used to screen for CVI but can also improve hemodynamics using FES-a portable theranostics application. Also, the implication of FES-fNIRS study raises an interesting hypothesis of increasing cerebral blood flow during the acute phase of the cerebrovascular accident, thus reducing the final infarct volume and improving the outcome. External counter pulsation has been shown to increase cerebral blood flow following a stroke [15]. Could FES using geko ${ }^{\mathrm{TM}}$ achieve a similar effect?

\section{REFERENCES}

[1] T. Hamaoka, K. K. McCully, M. Niwayama, and B. Chance, "The use of muscle near-infrared spectroscopy in sport, health and medical sciences: recent developments," Philos. Transact. A Math. Phys. Eng. Sci., vol. 369, no. 1955, pp. 4591-4604, Nov. 2011, doi: 10.1098/rsta.2011.0298.

[2] M. Ferrari, M. Muthalib, and V. Quaresima, "The use of near-infrared spectroscopy in understanding skeletal muscle physiology: recent developments," Philos. Transact. A Math. Phys. Eng. Sci., vol. 369, no. 1955, pp. 4577-4590, Nov. 2011, doi: 10.1098/rsta.2011.0230.

[3] M. Ferrari and V. Quaresima, "A brief review on the history of human functional near-infrared spectroscopy (fNIRS) development and fields of application," NeuroImage, vol. 63, no. 2, pp. 921-935, Nov. 2012, doi: 10.1016/j.neuroimage.2012.03.049.

[4] F. Scholkmann et al., "A review on continuous wave functional nearinfrared spectroscopy and imaging instrumentation and methodology," NeuroImage, vol. 85 Pt 1, pp. 6-27, Jan. 2014, doi: 10.1016/j.neuroimage.2013.05.004.

[5] S. Perrey and M. Ferrari, "Muscle Oximetry in Sports Science: A Systematic Review," Sports Med. Auckl. NZ, vol. 48, no. 3, pp. 597616, 2018, doi: 10.1007/s40279-017-0820-1.

[6] A. Dutta, R. Kobetic, and R. J. Triolo, “Ambulation after incomplete spinal cord injury with EMG-triggered functional electrical stimulation," IEEE Trans. Biomed. Eng., vol. 55, no. 2 Pt 1, pp. 791794, Feb. 2008, doi: 10.1109/TBME.2007.902225.

[7] A. Villringer, J. Planck, C. Hock, L. Schleinkofer, and U. Dirnagl, "Near infrared spectroscopy (NIRS): a new tool to study hemodynamic changes during activation of brain function in human adults," Neurosci. Lett., vol. 154, no. 1-2, pp. 101-104, May 1993.

[8] T. Yamaki, M. Nozaki, H. Sakurai, K. Soejima, T. Kono, and A. Hamahata, "Advanced chronic venous insufficiency is associated with increased calf muscle deoxygenation," Eur. J. Vasc. Endovasc. Surg. Off. J. Eur. Soc. Vasc. Surg., vol. 39, no. 6, pp. 787-794, Jun. 2010, doi: 10.1016/j.ejvs.2010.01.031.

[9] T. J. Huppert, S. G. Diamond, M. A. Franceschini, and D. A. Boas, "HomER: a review of time-series analysis methods for near-infrared spectroscopy of the brain," Appl. Opt., vol. 48, no. 10, pp. D280-D298, Apr. 2009.

[10] S. Jahani, S. K. Setarehdan, D. A. Boas, and M. A. Yücel, "Motion artifact detection and correction in functional near-infrared spectroscopy: a new hybrid method based on spline interpolation method and Savitzky-Golay filtering," Neurophotonics, vol. 5, no. 1, Jan. 2018, doi: 10.1117/1.NPh.5.1.015003.

[11] J. C. Ye, S. Tak, K. E. Jang, J. Jung, and J. Jang, "NIRS-SPM: statistical parametric mapping for near-infrared spectroscopy," NeuroImage, vol. 44, no. 2, pp. 428-447, Jan. 2009, doi: 10.1016/j.neuroimage.2008.08.036.

[12] C. M. Aasted et al., "Anatomical guidance for functional near-infrared spectroscopy: AtlasViewer tutorial," Neurophotonics, vol. 2, no. 2, Apr. 2015, doi: 10.1117/1.NPh.2.2.020801.

[13] M. Griffin, A. N. Nicolaides, D. Bond, G. Geroulakos, and E. Kalodiki, "The efficacy of a new stimulation technology to increase venous flow and prevent venous stasis," Eur. J. Vasc. Endovasc. Surg. Off. J. Eur. Soc. Vasc. Surg., vol. 40, no. 6, pp. 766-771, Dec. 2010, doi: 10.1016/j.ejvs.2010.06.019.

[14] M. C. Cheung, "Hemodynamics due to calf muscle activitybiophysical modeling and experiments using frequency domain near infrared spectroscopy in healthy humans," Thesis, State University of New York at Buffalo, 2018.

[15] R. M. Applebaum et al., "Sequential external counterpulsation increases cerebral and renal blood flow," Am. Heart J., vol. 133, no. 6, pp. 611-615, Jun. 1997, doi: 10.1016/s0002-8703(97)70161-3. 